\title{
Estudo do Seeing através da FWHM (Full Width at Half Maximum)
}

\author{
$\underline{\text { José Henrique Costa Pinto Souza }}{ }^{1}$, Paulo César da Rocha Poppe ${ }^{2}$ \\ 1. Bolsista Voluntário, Graduando em Física, Universidade Estadual de Feira de Santana, \\ e-mail: henriquecpsouza@yahoo.com.br. \\ 2.Orientador, Departamento de Física, Universidade Estadual de Feira de Santana, \\ e-mail: paulopoppe@uefs.com
}

PALAVRAS-CHAVE: Seeing, FWHM, turbulência.

\section{INTRODUÇÃO}

Nas áreas experimentais, condições do ambiente são de extrema importância para uma boa execução do experimento. Na Astronomia, como uma ciência observacional, esse aspecto não é diferente, pois a mesma possui uma dependência extrema de fatores como o clima, altitude, fases da Lua, turbulências atmosféricas, entre outros. Levando isso em consideração, são discutidas várias formas para determinar a qualidade de um sitio observacional, empregando técnicas simples ou completamente automatizadas. Nesse estudo, abordamos uma metodologia bastante simplificada baseada na análise de uma estrela observada em diferentes massas de ar. O resultado esperado é uma medida do "seeing", caracterizado como um fenômeno que se manifesta nas imagens formadas por telescópios ópticos instalados na superfície terrestre. Sua ação faz com que a imagem de uma estrela seja alargada em decorrência dos movimentos turbulentos da atmosfera terrestre. O "seeing" é uma medida em segundos de arco da largura máxima a meia altura (do inglês Full Width at Half Maximum $(F W H M)$ ), de um objeto (no caso uma estrela), o qual nos fornece uma ideia da turbulência atmosférica do local (sítio de observação). Considerando o brilho de uma estrela , quando os raios de luz provenientes desta fonte penetram na atmosfera, encontram um meio no qual as condições físicas (densidade, temperatura, pressão, iluminação) e de composição química ( percentagem relativa de gases)variam espacial e temporalmente(Harwit,1983). Em foco de um grande telescópio os efeitos da turbulência da atmosfera causam rapidamente imagens borradas (Pereyra 2003).

\section{MATERIAIS E METODOS}

Foram utilizados os telescópios CDK20 e Meade LX200 para a coleta das imagens nas cidades de Feira de Santana e Tanquinho, respectivamente. Inicialmente, com todos os instrumentos ligados e a CCD (Charge Coupled Device) com uma temperatura estabilizada $\left(\sim 5^{\circ} \mathrm{C}\right)$, identifica-se uma estrela alvo não muito brilhante (mag $\left.\sim 5\right)$ e aponta o telescópio para a realização do foco. Uma vez realizado, começamos a coleta de dados apontando para uma estrela no leste próxima ao horizonte. Essa estrela será observada ao longo da noite em diferentes massas de ar, e servirá para estimar o "seeing" do local. Obviamente, o tempo de integração adequado (em função da magnitude da estrela) é estimado em tempo real, de modo que não tenhamos saturadas, as quais são inúteis para o trabalho proposto.

$\mathrm{Na}$ parte da análise dos dados, empregamos o software "IRAF" (Image Reduction and Analysis Facility - http://iraf.noao.edu/), que reúne uma ampla coleção de softwares para uso 
geral em redução e análise de dados científicos. O projeto IRAF foi desenvolvido e é mantido pelo National Optical Astronomy Observatory, em Tucson, Arizona, nos Estados Unidos.

Empregamos também o visualizador de imagens DS9 (http://ds9.si.edu/site/Home.html), que representa uma poderosa ferramenta para analisar as imagens coletadas. A Figura 1 exemplifica esse aspecto para a estrela V474 Mon (alfa $=05^{\mathrm{h}} 59^{\mathrm{m}} 01^{\mathrm{s}}$, delta $=-09^{\circ} 22^{\prime} 5^{\prime}$, , J2000,0; B-V = 0,29 ,mag), observada em 17/12/2017 e usada para exemplificar esse estudo.

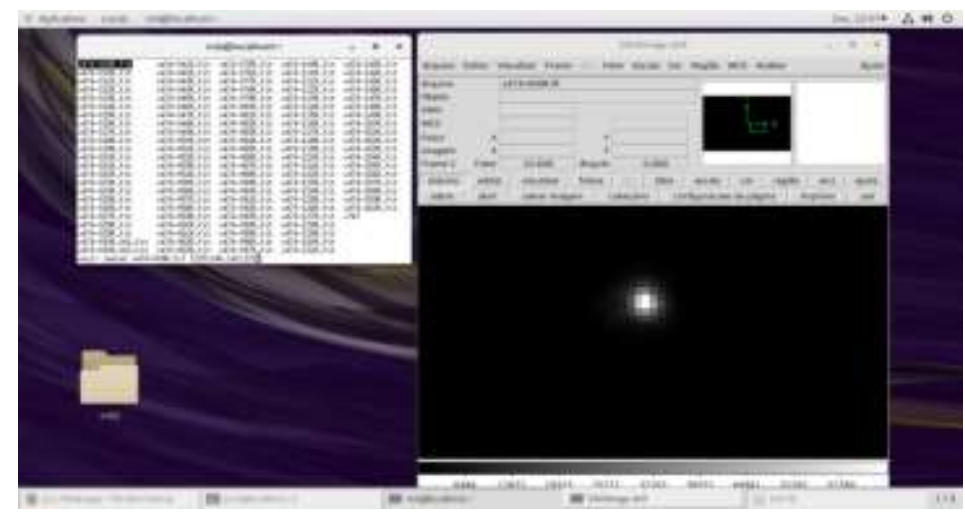

Figura 1. Programas utilizados (IRAF e DS9).

É necessário que seja selecionada uma região para obter o numero de contagens do céu de fundo. Com o comando imstat \{nome da imagem\} [região], selecionamos uma área de 21 pixeis quadrados próximos à estrela (figura 2).

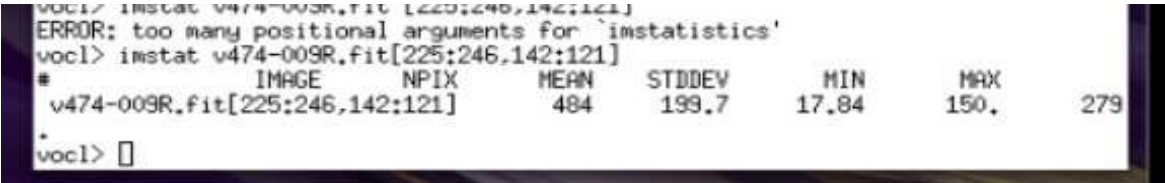

Figura 2. Comando utilizado para obter contagens do céu de fundo.

Pode se observar que a média de contagens equivale a 199,7, "um pouco deslocado da sua posição real". Usando a opção "tabela de pixeis" no DS9 e posicionando o cursor do mouse em cima da estrela (Figura 3), identificamos o pixel de maior intensidade e a intensidade dos outros.

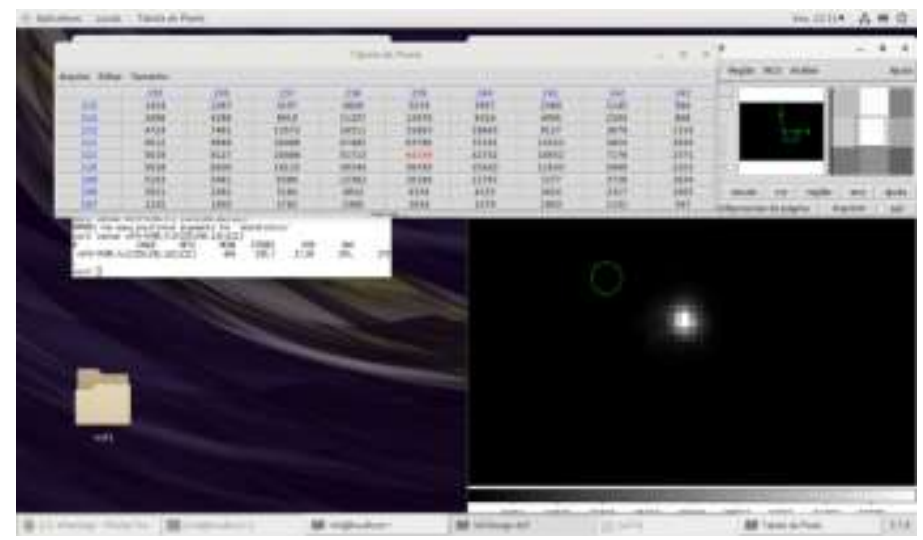

Figura 3.Tabela de pixeis. 


\section{RESULTADOS E DISCUSSÃO}

Com as imagens adquiridas, usamos o IRAF para encontrar o pixel de maior intensidade e a intensidade do céu de fundo, o qual é o valor é a metade da intensidade total da estrela, no nosso caso contamos os pixeis que superam esse valor. Ao encontrarmos o FWHM em relação ao diâmetro dos pixeis, precisamos converter esse valor para segundos de arco, ou seja, o valor do seeing é obtido em segundos de arco.

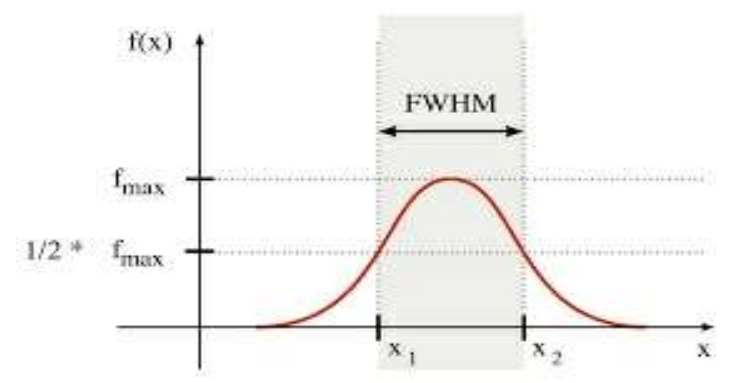

Figura 4. FWHM; Fonte: http://cosmobook.com.br/files/aula6.pdf

Na figura 5 podemos ver claramente a distribuição gaussiana da estrela com o comando imexamine posicionando o cursor na estrela e usando a tecla s.

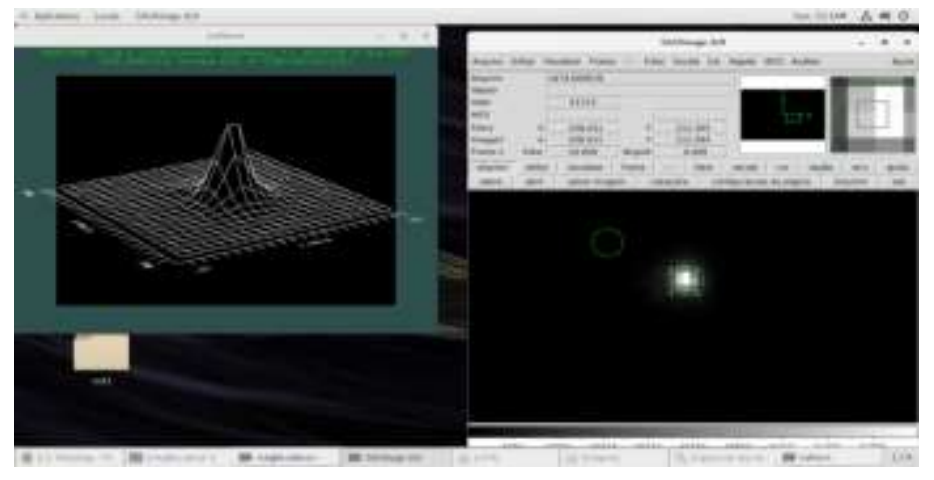

Figura 5. Plot 3d da distribuição da estrela.

A partir dos dados coletados em campo podemos construir um diagrama entre o horário e o seeing, Figura 6, para podermos ter uma melhor compreensão do quanto o mesmo varia durante uma noite.

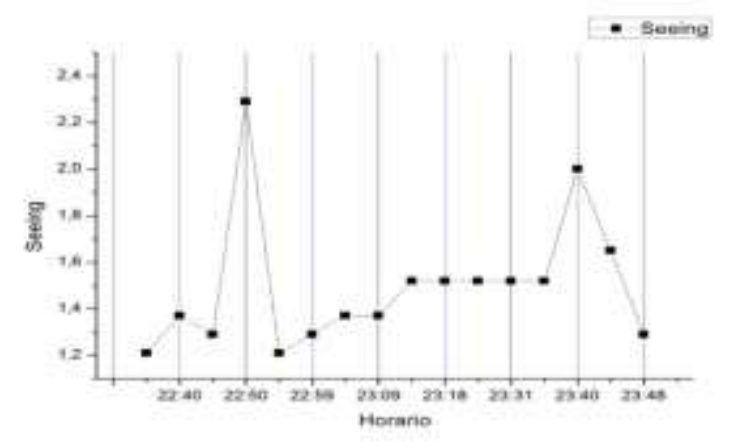

Figura 6. Diagrama do seeing em relação ao horário. 
Como o seeing é uma espécie de "espalhamento" da luz na imagem quanto menor seu valor melhor é a qualidade do céu. Podemos ver na figura 7 uma relação entre os valores de seeing e as condições do céu.

\begin{tabular}{|c|c|c|c|c|c|c|c|c|c|}
\hline \multicolumn{3}{|c|}{ Condiciones de malas a medianas } & \multicolumn{4}{|c|}{$\begin{array}{l}\text { Escala Pickering cotas en arc.seg. } \\
\text { de medianas a buenas de buenas a excelentes }\end{array}$} & \multicolumn{3}{|c|}{ de excelentes a optimas } \\
\hline 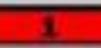 & 2 & 3 & 4 & 5 & 6 & 7 & 8 & 9 & 10 \\
\hline \multicolumn{10}{|c|}{ Valores del SEEING on su punto FWHM, para cada nivel de la escala } \\
\hline 13,19 & 10,21 & 7,91 & 6,12 & 4,74 & 3,67 & 2,84 & 2,20 & 1,70 & 1,32 \\
\hline
\end{tabular}

Figura 7. Fonte:www.invlumer.e.telefonica.net/Planificando/SEEING/Escala_SEEING.htm

Para encontrar o intervalo de confiança das medidas fazemos uso da equação:

$$
I C=S_{m e d} \pm Z \frac{\sigma}{\sqrt{n}}
$$

Onde $\sigma$ é o desvio padrão ,n é o numero de amostras e para um intervalo de confiança de $95 \%$ $\mathrm{Z}$ é igual a $1.96, \operatorname{logo} 1.381 \geq$ seeing $\geq 1.652$.

\section{CONCLUSÃO}

Embora seja possível realizar estudos fotométricos para objetos relativamente brilhantes (por conta da alta poluição luminosa presente na cidade de Feira de Santana), estamos empenhados em escolher um novo sítio observacional para acomodar o telescópio CDK20 e a instrumentação associada. Um local muito promissor foi revelado na zona rural de Tanquinho ( 1 hora) do Observatório Astronômico Antares.

Várias visitas técnicas já foram realizadas em épocas diferentes do ano para perceber visualmente e com o telescópio MEADE LX200 $(25 \mathrm{~cm})$ a qualidade do céu. No entanto, com o uso da CCD, tivemos problemas para a aquisição das imagens. Algumas delas foram obtidas com um pequeno sensor LPI (Lunar-Planet Image), mas como o software associado não permitia controlar o tempo de exposição, todas as imagens foram saturadas, impossibilitando um estudo preliminar. O problema já foi corrigido e uma nova missão será realizada nos próximos meses para mensurar e comparar a qualidade do céu da zona rural de Tanquinho com aquela da cidade de Feira de Santana. No entanto, este estudo foi importante para uma primeira compreensão da técnica, do uso do telescópio e dos possíveis problemas associados às observações no óptico.

\section{REFERENCIAS}

PEREYRA, Antonio; BAELLA, Nobar. Medidas de seeing en el Observatorio de Huancayo. REVCIUNI, v. 7, n. 1, p. 103, 2003.

SCHÖNGARTH, Roberto. Metodología para cálculo de seeing en el Observatorio Astronómico.Centroamericano de Suyapa, Tegucigalpa, Honduras. Ciencias Espaciales, v. 1, n. 1, 2011.

HARWIT,M. 1983. Astrophysical concepts, Wiley ,New York 